\title{
Exon Arrays Reveal Alternative Splicing Aberrations in Parkinson's Disease Leukocytes
}

\author{
Lilach Soreq $^{a}$ Hagai Bergman ${ }^{a, c, d}$ Zvi Israel $^{d}$ Hermona Soreq ${ }^{b, c}$ \\ ${ }^{a}$ Department of Medical Neurobiology, Hebrew University-Hadassah Faculty of Medicine, ${ }^{b}$ Department of \\ Biological Chemistry, The Life Sciences Institute, and ' The Edmond and Lily Safra Center for Brain Sciences, \\ The Hebrew University of Jerusalem, and ${ }^{\mathrm{d}}$ Center for Functional and Restorative Neurosurgery, Department of \\ Neurosurgery, Hadassah University Hospital, Jerusalem, Israel
}

\section{Key Words}

Alternative splicing • Exon microarrays $\cdot$ Leukocytes •

Parkinson's disease $\cdot$ Risk markers alternative splicing of numerous transcripts. The aberrant alternative splicing in PD patients' blood cells has potential implications for early diagnosis and future therapeutics.

Copyright $\odot 2011$ S. Karger AG, Basel

\begin{abstract}
Background: Parkinson's disease (PD) is the second most frequent neurodegenerative disease worldwide. Clinical diagnosis can only be made when the vast majority of the dopaminergic cell population has died. However, the cause(s) for sporadic PD is/are yet unclear. Transcript changes have recently been described in PD patients' whole blood cells, but corresponding splicing patterns remained unknown. Objective: To search for alternative splicing aberrations in PD patients' blood leukocytes. Methods: We applied exon microarrays to profile PD patients' blood leukocyte mRNA. Exon level splicing analysis served as a basis for downstream classification and functional analyses. Results: Patients and carefully matched controls were classified by the splicing exon profiles of their leukocyte transcripts. Specifically, many exons were downregulated in PD patients compared to controls. Functional analysis highlighted aberrant splicing of PDrelated transcripts and impaired NF- $\kappa$ B cascade and immune response. Conclusion: PD patient's blood leukocytes exhibit
\end{abstract}

\section{Introduction}

Parkinson's disease (PD) is the most prevalent neurodegenerative condition that involves motor symptoms [1]. Unlike Alzheimer's disease, its pathology is distributed throughout the entire nervous system [2]. PD has a prevalence of approximately $0-1 \%$ among individuals $65-69$ years of age, rising to $1-3 \%$ among those older than 80 years [3] and $4-5 \%$ above 85 [4]. PD prevalence is predicted to grow worldwide within the next decades [5]. Six genes have been implicated in the less frequent, early appearance, genetic form of the disease (SNCA, Parkin, PINK1, DJ1, LRRK2 and GBA). However, the genes and mechanisms that underlie the neuronal degeneration in the majority of cases, sporadic PD, are still unknown. Furthermore, by the time a diagnosis of $\mathrm{PD}$ is made following the onset of clinical symptoms, approximately $80 \%$ of the striatal dopaminergic neurons may have already died. Al-

\section{KARGER}

Fax +4161306 1234

E-Mail karger@karger.ch

www.karger.com
C 2011 S. Karger AG, Basel

$1660-2854 / 12 / 0104-0203 \$ 38.00 / 0$

Accessible online at:

www.karger.com/ndd
Hermona Soreq, $\mathrm{PhD}$

Department of Biological Chemistry

The Hebrew University of Jerusalem, The Edmond J. Safra Campus

Givat Ram, Jerusalem 91904 (Israel)

Tel. +972 2658 5109, E-Mail soreq@cc.huji.ac.il 
Fig. 1. (a) Study participants' clinical variables. (b) Alternatively spliced exons discriminate PD patients from controls. Splicing index values normalized to the constitutive gene level expression served to classify the samples using hierarchical classification. PD patients were distinguished from control subjects correctly based on the normalized splicing index values of the 163 alternatively spliced probe sets. (c) The most prominent alternatively spliced events in patients compared to controls (SI Benjamini-Hochberg corrected FDR $\mathrm{p}<0.005,<2$-fold change) were detected in 18 distinct genes exons, exhibiting more downregulated exons.

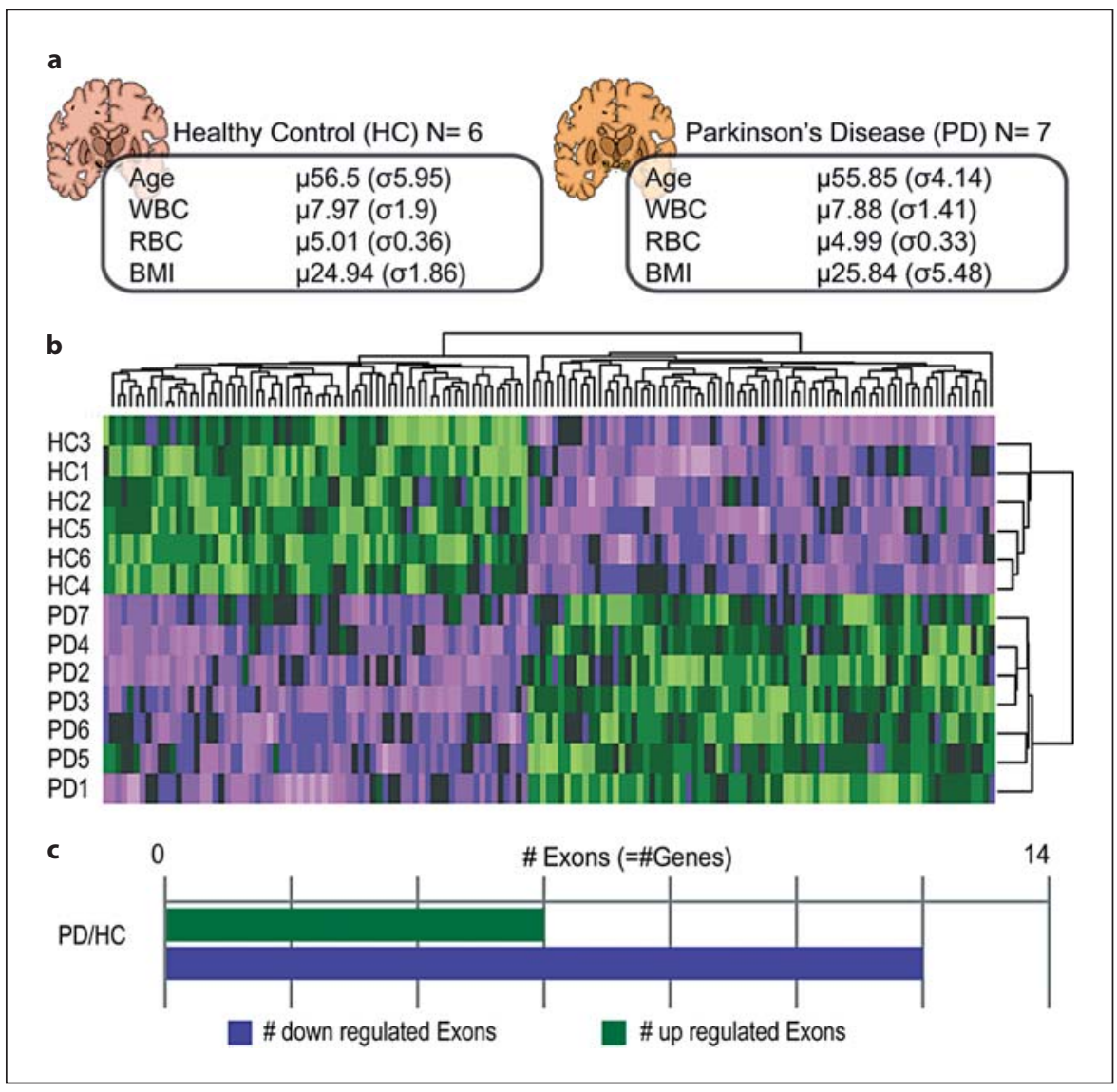

ternative RNA splicing is the major mechanism to increase functional diversity given the limited number of human genes [approx. 25,000 as per current Ensembl DB version (v64)]. About $92-94 \%$ of all human protein-coding genes undergo alternative splicing to produce more than one isoform [6]. Here, we report massive splicing changes in PD blood cells. Alternative splicing is involved in several human diseases including cancer development $[7,8]$. In sporadic neurodegeneration, however, its involvement awaits further studies.

We previously found pronounced changes in multiple transcripts from whole blood nucleated cells of PD patients compared to healthy and neurological controls by analyzing $3^{\prime}$ microarray datasets of others $[9,10]$.

\section{Methods}

This study was authorized and approved by the Ethics Committee of the Human Review Board (Hadassah University Hospital, Ein-Kerem, approval 6-07.09.07) in accordance with the
Declaration of Helsinki principles. Following oral agreement, all participants signed informed consent. Blood samples were taken from 7 advanced PD male patients and 6 healthy agematched male control subjects who were assessed for their clinical background and state and filled out detailed medical history questionnaires. RNA was extracted from LeukoLock filters (Ambion, Applied-Biosystems, Inc., Foster-City, Calif., USA) and hybridized to Affymetrix GeneChip ${ }^{\circledR}$ Exon_1.0_ST_Array (Affymetrix, Santa Clara, Calif., USA) microarrays according to the manufacturer's instructions. The open-source AltAnalyze program (version 2.03, using Ensembl version 62) served for exon level analysis and Matlab R20011A (7.12.0) for downstream analyses (false discovery rate calculations and clustering analysis).

\section{Results}

Leukocyte mRNA from 7 carefully matched PD patients and 6 healthy controls (fig. 1a) was examined using exon microarrays. Advanced alternative splicing analysis served to search for disease-characteristic transcript changes. 
Table 1. Alternatively spliced exons in PD patients compared to controls

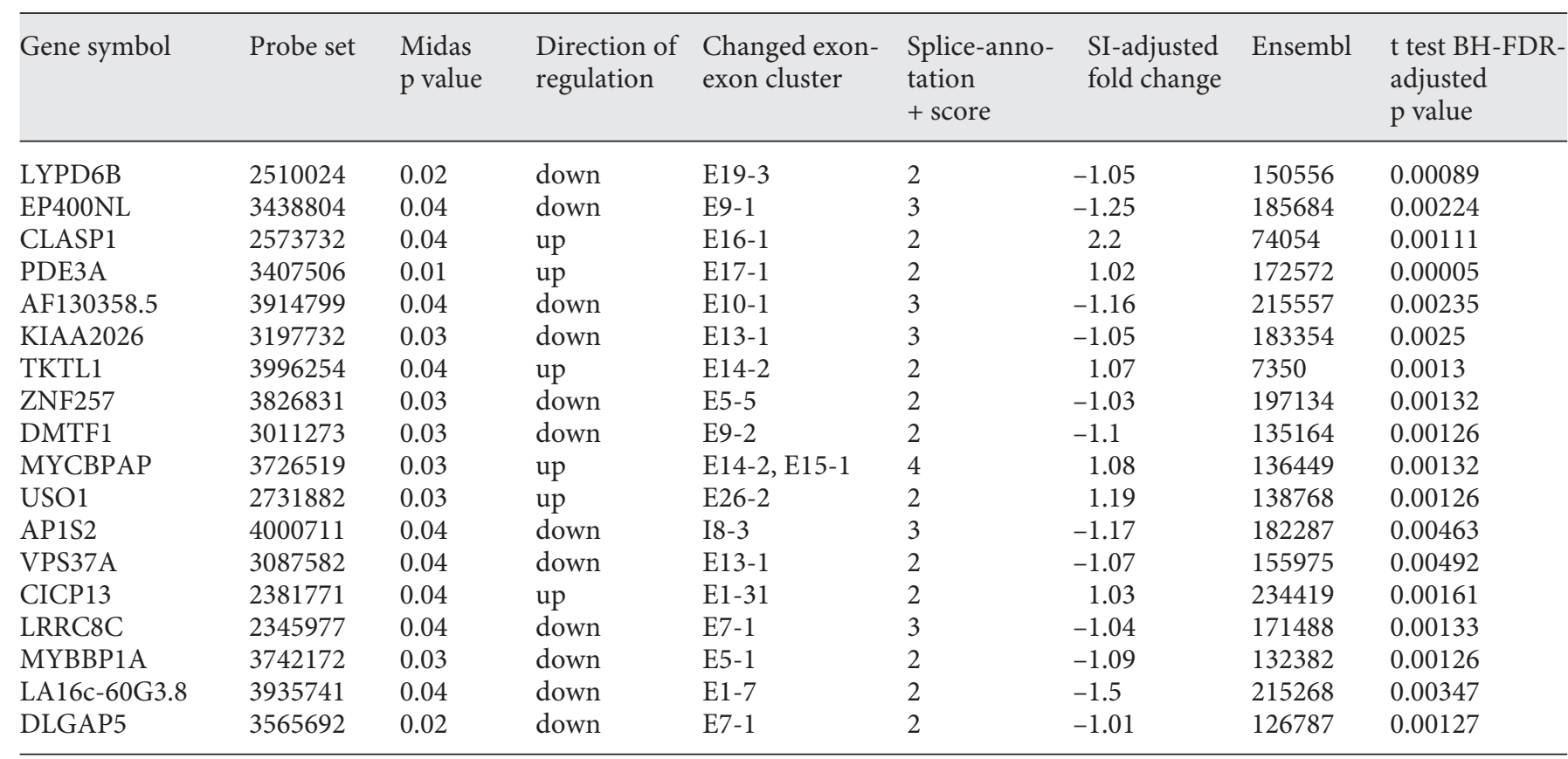

Listed are the alternative splicing events detected by core probe sets exhibiting a log ratio between tissues and having an SI FDRadjusted $p$ value $<0.005$ and a detection above background $\mathrm{p}$ value $<0.05$ for PD patients compared to matched healthy control subjects. $\mathrm{BH}-\mathrm{FDR}=$ Benjamini and Hochberg false discovery rate.

\section{Differential Splicing Patterns in PD Patients'}

Compared to Control Leukocytes

The expression profiles of PD patients' leukocytes were compared to those of age- and gender-matched healthy control volunteers. $t$ test on splicing index (SI) calculations detected 163 alternative splicing events in 150 distinct genes (SI t test $\mathrm{p}$ value $<0.05$ and detection above background $p$ value $<0.05$ ). All the detected genes passed the Benjamini and Hochberg false discovery rate test [11]. Hierarchical classification separated patients from controls based on the gene-normalized SI values (fig. 1b). The classification was conducted blinded to the clinical condition, using Euclidean distance for both rows (exons) and columns (samples).

Highly alternatively spliced exons with a log ratio between tissues (2-fold SI change) having an SI p value $<0.005$ and a detection above background $p$ value $<0.05$ entailed 18 events in 18 distinct genes, including the leucine-rich repeat containing LRRC8C and the ATP-binding (AF130358.5) gene (table 1). Post hoc functional enrichment analysis using EASE [12] indicated functional involvement of the detected genes in alternative splicing and protein transport.

Parkinson's Leukocyte Alternative Splicing

\section{Discussion}

Global inspection revealed altered alternative splicing profiles in PD patients compared to healthy controls. To our knowledge, this is the first study that examines alternative splicing on a global scale in PD patients.

PD patients exhibited mainly decreases in the most prominent 18 detected exon inclusion events as compared with healthy control subjects. The prominently changed transcripts included an ATP-binding (AF130358.5) gene and a $\mathrm{PD}$-associated leucine-rich repeat family member (LRRC8C). Additional detected events belonged to genes which participate in the alternative splicing process. The PD patient participants in this study were selected among those nominated for deep brain stimulation neurosurgery (i.e. bilateral implantation of deep brain stimulators into the subthalamic nucleus), which naturally limited the group size yet further allowed us to test self-controlled deep brain stimulation-induced changes. In a continuation study, each participant was tested 1 day prior to neurosurgery, and approximately 3 months after deep brain stimulation (upon symptom stabilization). Changes occurred in alternative splicing processes, in disease- 
related transcripts (such as SNCA and PARK7) and in neuroinflammatory processes. Notably, $13 \%$ of the transcripts changed in PD were subsequently changed following deep brain stimulation and the neuroinflammatory processes that were impaired in PD were decreased following deep brain stimulation [13]. Future studies on larger cohorts will enable to pinpoint those exons that can serve for disease diagnostics and identify variant-specific targets for splice switching therapies.

\section{Acknowledgements}

We thank Dr. Nathan Salomonis (Gladstone Institute of Cardiovascular Disease; San Francisco, Calif., USA) for helpful discussions. This study was funded by the Thyssen Foundation (to H.B. and H.S.), and the Rosetrees Foundation (to H.S.), and the Gatsby Charitable Foundation (to Z.I.).

\section{References}

$\checkmark 1$ Nussbaum RL, Ellis CE: Alzheimer's disease and Parkinson's disease. N Engl J Med 2003; 348:1356-1364.

2 Braak H, Del Tredici K: Invited article: nervous system pathology in sporadic parkinson disease. Neurology 2008;70:1916.

-3 Tanner CM, Goldman SM: Epidemiology of Parkinson's disease. Neurol Clin 1996;14: 317-335.

4 Fahn S: Description of Parkinson's disease as a clinical syndrome. Ann NY Acad Sci 2003; 991:1-14.

5 Chopra V, Fox JH, Lieberman G, Dorsey K, Matson W, Waldmeier P, Housman DE, Kazantsev A, Young AB, Hersch S: A smallmolecule therapeutic lead for Huntington's disease: preclinical pharmacology and efficacy of $\mathrm{C} 2-8$ in the R6/2 transgenic mouse. Proc Natl Acad Sci USA 2007;104:1668516689.
-6 Wang ET, Sandberg R, Luo S, Khrebtukova I, Zhang L, Mayr C, Kingsmore SF, Schroth GP, Burge CB: Alternative isoform regulation in human tissue transcriptomes. Nature 2008;456:470-476.

7 Guo X, Chen QR, Song YK, Wei JS, Khan J: Exon array analysis reveals neuroblastoma tumors have distinct alternative splicing patterns according to stage and MYCN amplification status. BMC Med Genomics 2011;4: 35.

8 Prinos P, Garneau D, Lucier JF, Gendron D, Couture S, Boivin M, Brosseau JP, Lapointe E, Thibault P, Durand M, Tremblay K, Gervais-Bird J, Nwilati $H$, Klinck R, Chabot B, Perreault JP, Wellinger RJ, Elela SA: Alternative splicing of SYK regulates mitosis and cell survival. Nat Struct Mol Biol 2011;18:673679.

9 Scherzer CR, Eklund AC, Morse LJ, Liao Z, Locascio JJ, Fefer D, Schwarzschild MA, Schlossmacher MG, Hauser MA, Vance JM, Sudarsky LR, Standaert DG, Growdon JH, Jensen RV, Gullans SR: Molecular markers of early Parkinson's disease based on gene expression in blood. Proc Natl Acad Sci USA 2007;104:955-960.
10 Soreq L, Israel Z, Bergman H, Soreq H: Advanced microarray analysis highlights modified neuro-immune signaling in nucleated blood cells from Parkinson's disease patients. J Neuroimmunol 2008;201-202:227236.

11 Benjamini Y, Hochberg Y: Controlling the false discovery rate: a practical and powerful approach to multiple testing. J R Stat Soc 1995;57:289-300.

-12 Huang da W, Sherman BT, Lempicki RA: Systematic and integrative analysis of large gene lists using DAVID bioinformatics resources. Nat Protoc 2009;4:44-57.

13 Soreq L, Bergman H, Goll Y, Greenberg DS, Israel Z, Soreq H: Deep brain stimulation induces rapidly reversible transcript changes in Parkinson's leukocytes. J Cell Mol Med 2011, E-pub ahead of print, 2011 Sep 12. DOI: $\underline{10.1111 / \mathrm{j} .1582-4934.2011 .01444 . \mathrm{x}}$ 